\title{
The Research and Analysis of One New Deep-sea Releaser
}

\author{
Xiao Hua Mou ${ }^{1, a}$, Zhe $\mathrm{Xu}^{2, b^{*}}$ and Peng Wei ${ }^{3, \mathrm{c}}$ \\ ${ }^{2}$ Room419, College of Eng. Sci. Tech., Shanghai Ocean University, No.999, Hu Cheng Huan Road, \\ Lin gang new city, Shanghai, P.R. China \\ ${ }^{1,3}$ Room222, College of Eng. Sci. Tech., Shanghai Ocean University, No.999, Hu Cheng Huan \\ Road, Lin gang new city, Shanghai, P.R. China \\ aa0715002009@163.com, b734529312 @qq.com, '1378744523@qq.com
}

Keywords: releaser; analysis of structure strength; dynamic simulation

\begin{abstract}
Deep-sea releaser is very key element in the research of deep-sea scientific and has broad applications, the most typical releaser is the acoustic releaser. This paper expounds one new type releaser on the principle of lever to accomplish the purpose of reducing the failure rate of release making full use of limited space and it has simple structure and easy to operate. By using the software of ANSYS and Adams, obtained the relevant key data after analyzing to its structure strength and dynamic simulation so as to further demonstrate the practicability and reliability of this release. There is great significance about the conclusion to further advance and implement the marine strategy.
\end{abstract}

\section{Introduction}

Deep-sea releaser is very key element in the research of deep-sea scientific and has broad applications, the most typical releaser is the acoustic releaser, the literature [1] introduces the current research status and development situation of international deep-sea acoustic releaser and pointed out that our country still has big gap compared with foreign advanced level, following by electromagnetic releaser and so on. Because the deep investigation may be failed due to the volume of a vehicle or other scientific instruments, will produce larger buoyancy, so you need to release suspension larger weight in order to overcome the effects of buoyancy, but when the weight to be a certain extent may be can't release weights due to the water or their own reasons of releaser and so on, which can lead to be lost of valuable instrument so that having a huge loss. Therefore, the check and ratify release load is bigger, the better of the releaser in the certain cases and the space for approval of releasing.

The release mechanism is studied in this paper that can be made for acoustic releaser controlled by release device, can also be made for electromagnetic releaser controlled by release timing. This study is through the three-dimensional modeling software by multi-body dynamics method to establish the dynamic model of releaser, according to the MORISION equation and the unilateral model to analyze the release of hydrodynamic and contact force, analyzing the static analysis of the structure with the ANSYS software, releasing on the process of dynamic simul- ation in Adams by the numerical method, and analyzing the motion state of release structure.

\section{The Work Principle and Dynamics Calculation of Deep-sea Releaser}

\section{The Work Principle of Deep-sea Releaser}

Due to the work environment of deep-sea releaser is generally worse and most of its release is through long-range acoustic release or electromagnet released regularly, so releaser should be designed with simple structure and easy to operate, thus reducing the failure probability of release. Figure 1 is the appearance of the deep-sea releaser designed for this article research, the working depth can reach $11000 \mathrm{~m}$, the load capacity can reach more than $5000 \mathrm{~kg}$. The main internal structure of release mechanism as shown in figure 2.The work process is: the latch will pull up when the water computer launches a long-range signal or at the end of the electromagnet timing, hook 2 will produces clockwise rotation because of the pressure produced by its own gravity and hung on 
anchor weight indirectly by hook 1 when latch completely off the hook 2, hook 1 will be also counterclockwise rotation at this time because of the clockwise rotation of hook 2, thus to release the bolt and carrying heavy objects, accomplish the work of release and achieve smooth submersible underwater operation or buoyancy.

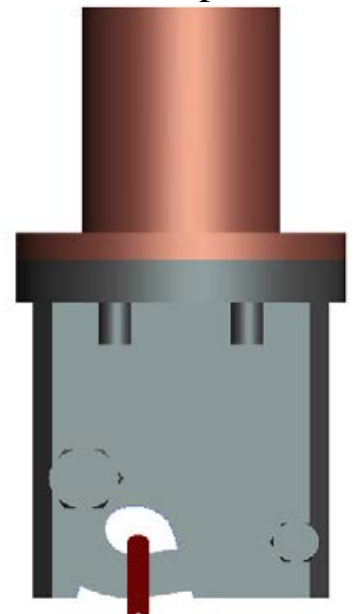

Fig. 1 The appearance of releaser

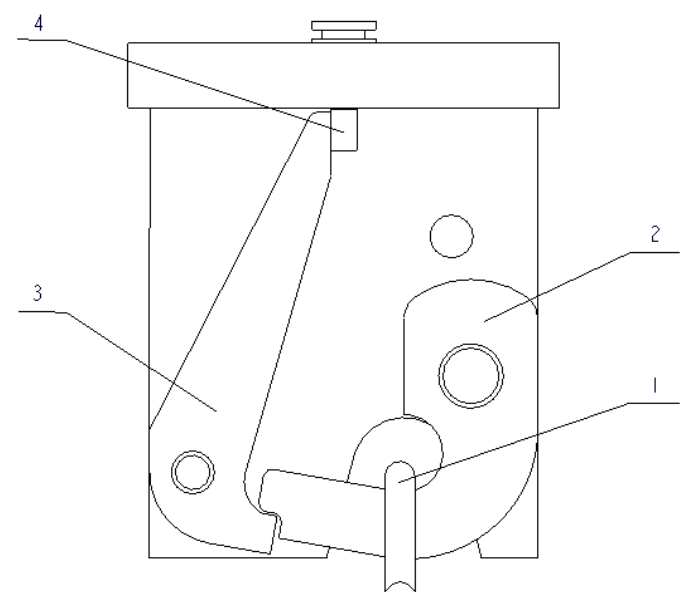

1-Anchor link 2-Hook 1 3-Hook 2 4-Latch

Fig. 2 The release mechanism of releaser

\section{The Contact Force Model Between of Components}

There are more complex relationship of contact between each component of the release mechanism that shown in the figure 2: there are some phenomena in the process of working between latch and the hook 2, hook1 and the hook 2, hook1 and anchor rod, hook and support shaft, such as contact, away and collision phenomenon. There is the following relationship between contact normal force $\mathrm{P}$ and deformation $\delta$ based on Hertz contact theory $[5,6]$ :

$\delta=\frac{\mathrm{a}^{2}}{\mathrm{R}}=\left(\frac{9 \mathrm{P}^{2}}{16 \mathrm{RE}^{2}}\right)^{\frac{1}{3}}$.

among: $\frac{1}{\mathrm{R}}=\frac{1}{\mathrm{R}_{1}}+\frac{1}{\mathrm{R}_{2}} ; \frac{1}{\mathrm{E}}=\frac{\left(1-\mu_{1}^{2}\right)}{\mathrm{E}_{1}}+\frac{\left(1-\mu_{2}\right)^{2}}{\mathrm{E}_{2}}$.

then

$$
\mathrm{P}=\delta^{\frac{3}{2}} \sqrt{\frac{16 \mathrm{RE}^{2}}{9}} .
$$

According to $k=\sqrt{\frac{16 R E^{2}}{9}}$ be the equivalent stiffness, so contact normal force $\mathrm{P}$ :

$$
\mathrm{P}=\mathrm{k} \delta^{\frac{3}{2}} \text {. }
$$

According to unilateral contact model, considering the damping force and the normal force of the contact surface and material characteristic, we can get the general formula in contact and collision force of different components of releaser:

$$
F_{c}= \begin{cases}k \delta^{\alpha}+c \delta & \delta \geq 0 \\ 0 & \delta<0\end{cases}
$$

\section{The Structural Analysis and Simulation of Systematic Model}

\section{The Structural Statics Analysis of Systematic Model}

Put the model of releaser which have been built in the 3D modeling software pro/e import into structural strength analysis software ANSYS Workbench, and then added the corresponding relationship of contact and collision on the corresponding components and added the corresponding constraint and load after dividing the grid (add 5000N vertical downward force, instead of 
suspended load of $500 \mathrm{~kg}$ ), finally we could get the analytical cloud of the stress as shown in figure 3. We can find the maximum stress on the release is 1.6979 e08 Pa from the analytical cloud of stress, but the tensile yield strength of the stainless steel material is $2.07 \mathrm{e} 08 \mathrm{~Pa}$ which chosen by the model, not exceed the limit of yielding. And it can be seen that the stress of the latch is relatively small.

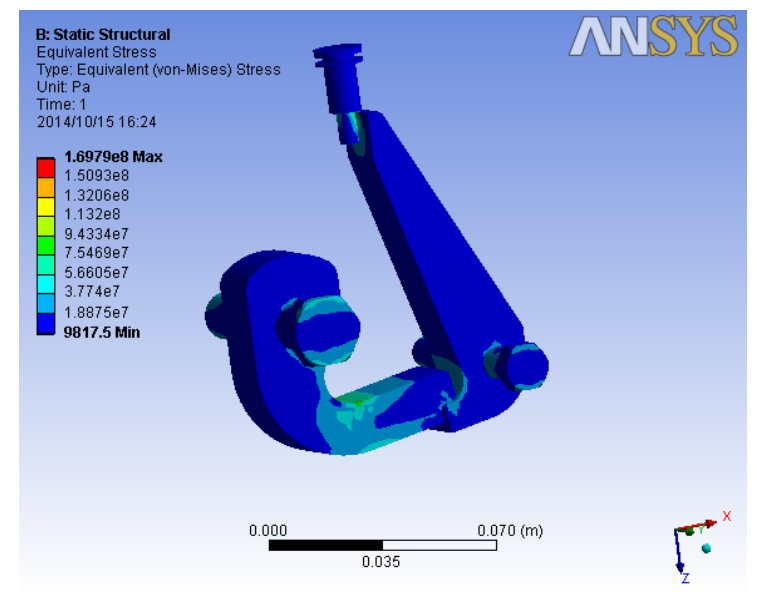

Fig. 3 The analytical cloud of stress

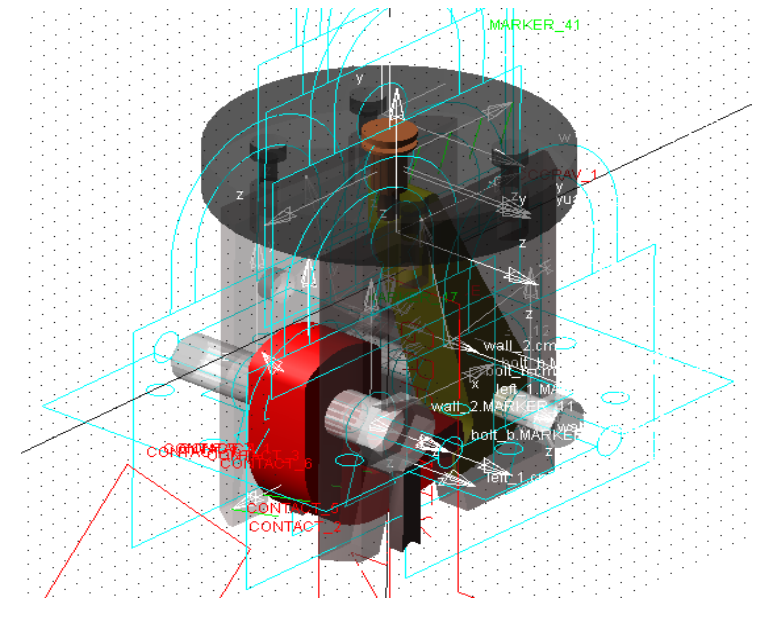

Fig. 4 Virtual prototype of releaser

\section{Dynamic Simulation of Systematic Model}

The dynamic model set up in Pro/e before was imported into the multi-body dynamics simulation software Adams, and adding force to the corresponding components according to the force function established before, establishing the virtual prototyping, as shown in figure 4, and adding corresponding force to latch according to the STEP function so that meet the requirements of the releasing process to force, so as to simulate the whole process of the release mechanism in Adams.

The angular acceleration curves of hook 1 and the weight of the $\mathrm{Y}$ direction displacement curves after finished the load rejection in different direction tilt when influenced by the flow of different direction in the process of release as shown in the figure 5 and figure 6 respectively, from the three graphs not only can see no matter under what kind of position, release mechanism would be all able to throw the weight load, and at the same time, the angular acceleration when tilted of the release mechanism is much less than the angular acceleration of release mechanism under the normal conditions in the instant of releasing the load although happened the inevitable collision to produce the angular acceleration during the process of falling of appliances under the relative conditions. Those proved that the release mechanism has the reliability of the underwater work under the bad environment.

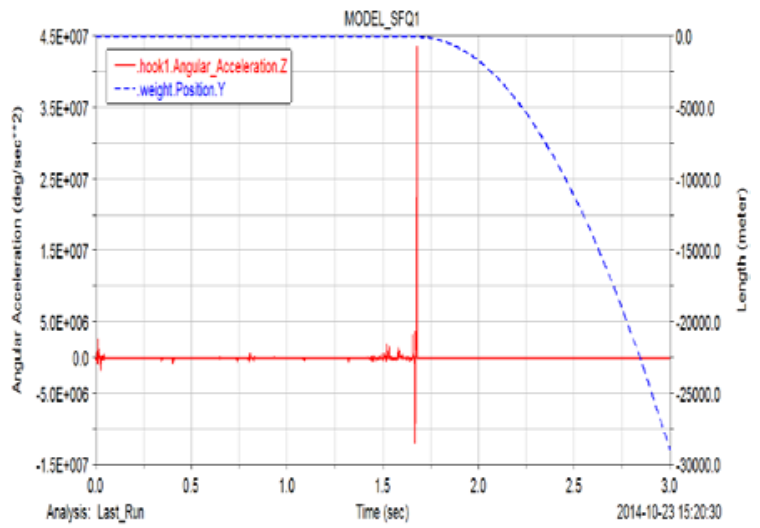

Fig. 5 The curves of angular acceleration and displacement of weight under normal release

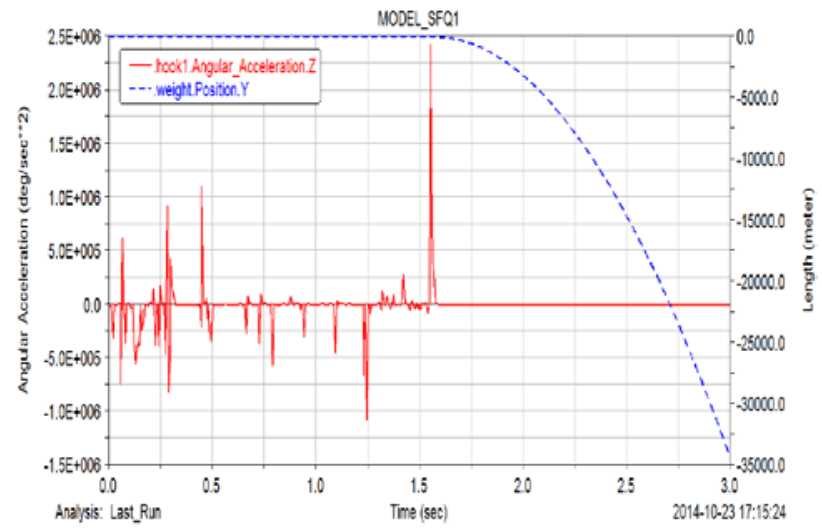

Fig. 6 The curves of angular acceleration and displacement of weight when leaning to the front or black of releaser 


\section{Summary}

In this paper, verified the feasibility of the release mechanism in detail and get the intensity and the deformation analytical cloud of each component with the motion parameters and trajectory through two analysis software ANSYS and Adams, proving the reliability and the practicability of the release mechanism. Not only plays an important role about this paper for the releaser in the future production or in the practical work, but also has great importance to improve our country's strength of scientific research in the deep-sea.

\section{Reference}

[1] X.Z. Chen, X.M. Nie, X. Chen. Technological development prospects of deep-sea release. Proceedings of 2008 ocean frontier technology BBS, Guangzhou: 2008.

[2] X. Wang. Production device design and test of CTD electronically controlled electromagnetic release mechanism in many bottles of water. Marine technology, 2003, 22 (12): 55-58.

[3] R.Q. Tang, Z.Y. Cao, X.X. Zhang. Development of the cast carrier system about the "explorer” autonomous underwater robot. Oceanographic engineering, 2001, 19 (2): 85-87.

[4] J.C. Duinker, A. Kortzinger, R.R.J. Phillips, et al. Recovery of deep-sea moorings with Mir submersibles. Sea Technology, 1994, 35(4): 34-42.

[5] D.P. Jin, H.Y. Hu. Collision and vibration control. Beijing: Science press, 2005.

[6] C.J. Fan, G.M. Xiong, M.F. Zhou. Virtual prototype software: Application and increase of ADAMS. China machine press, 2006. 